\title{
Spin-Boson and Spin-Fermion Topological Model of
}

\section{Consciousness}

\author{
Aibassov Yerkin ${ }^{1}$, Yemelyanova Valentina ${ }^{1}$, Nakisbekov Narymzhan ${ }^{1}$, Alzhan Bakhytzhan ${ }^{1}$ and Savizky Ruben ${ }^{2}$ \\ 1. Research Institute of New Chemical Technologies and Materials, Kazakh National University Al-Farabi, Almaty 005012, Kazakhstan \\ 2. Columbia University, 3000 Broadway, New York, NY, 10027, USA
}

Abstract: The authors propose a new approach to the theory of spin-boson and spin-fermion topological model of consciousness. The authors will offer a common mechanism of spin-boson and spin-fermion topological model of consciousness.

Key words: Spin-boson, spin-fermion, topology, model of consciousness, magnetic field.

\section{Introduction}

Recently, much attention is removed study of theory of consciousness [1-5]. All processes in the human brain occur in the form of electromagnetic processes. Therefore, it was interesting to see consciousness in terms of spin-boson and spin-fermion topological model.

The aim is to study the spin-boson and spin-fermion topological model of consciousness.

The novelty of the work lies in the fact that the authors have proposed a new mechanism of spin-boson and spin-fermion topological model of work of consciousness.

\section{Theory}

Neuronal membrane saturated carrier spin nuclei such as $1 \mathrm{H}, 13 \mathrm{C}$ and $31 \mathrm{P}[1,2]$. Neuronal membrane are the matrix of the brain electrical activity and play a vital role in the normal functions of the brain and conscious of their basic molecular components are phospholipids, proteins and cholesterol. Each phospholipid contains $1 \% 31 \mathrm{P}, 1.8 \% 13 \mathrm{C}$ and over $60 \% 1 \mathrm{H}$ lipid chain. Neuronal membrane proteins such as ion channels and receptors neural transmitters

Corresponding author: Aibassov Yerkin, professor, research field: metal organic chemistry of uranium and thorium, $\mathrm{As}, \mathrm{Sb}$ and $\mathrm{Bi}$. also contain large clusters spin-containing nuclei. Therefore, they are firmly convinced that the nature of the spin quantum used in the construction of the conscious mind. They suggested within neurobiology that perturbation anesthetics oxygen nerve pathways in both membrane proteins and may play a general anesthesia. Each $\mathrm{O}_{2}$ comprises two unpaired valence electrons strongly paramagnetic and at the same time as the chemically reactive bi-radical. It is able to produce large pulsed magnetic field along its path of diffusing. Paramagnetic $\mathrm{O}_{2}$ are the only breed can be found in large quantities in the brain to the same enzyme producing nitric oxide (NO). $\mathrm{O}_{2}$ is one of the main components for energy production in the central nervous system.

NO is unstable free radical with an unpaired electron and one recently discovered a small neural transmitter, well known in the chemistry of spin-field concentrated on the study of free radical-mediated chemical reactions in which very small magnetic energy conversion can change the non-equilibrium spin process. Thus, $\mathrm{O}_{2}$ and $\mathrm{NO}$ can serve as catalysts in a spin-consciousness associated with neuronal biochemical reactions such as the double paths reaction initiated by free radicals.

\section{Results and Discussion}

They present the following Postulates: (a) 
Consciousness is intrinsically connected to quantum spin; (b) The mind-pixels of the brain are comprised of the nuclear spins distributed in the neural membranes and proteins, the pixel-activating agents are comprised of biologically available paramagnetic species such as $\mathrm{O}_{2}$ and $\mathrm{NO}$, and the neural memories are comprised of all possible entangled quantum states of the mind-pixels; (c) Action potential modulations of nuclear spin interactions input information to the mind pixels and spin chemistry is the output circuit to classical neural activities; and (d) Consciousness emerges from the collapses of those entangled quantum states which are able to survive decoherence, said collapses are contextual, irreversible and non-computable and the unity of consciousness is achieved through quantum entanglement of the mind-pixels.

In Postulate (a), the relationships between quantum spin and consciousness are defined based on the fact that spin is the origin of quantum effects in both Bohm and Hestenes quantum formulism and a fundamental quantum process associated with the structure of space-time.

In Postulate (b), they specify that the nuclear spins in both neural membranes and neural proteins serve as the mind-pixels and propose that biologically available paramagnetic species such as $\mathrm{O}_{2}$ and $\mathrm{NO}$ are the mind-pixel activating agents. The authors also propose that neural memories are comprised of all possible entangled quantum states of mind-pixels.

In Postulate (c), they propose the input and output circuits for the mind-pixels. As shown in a separate paper, the strength and anisotropies of nuclear spin interactions through J-couplings and dipolar couplings are modulated by action potentials. Thus, the neural spike trains can directly input information into the mind-pixels made of neural membrane nuclear spins. Further, spin chemistry can serve as the bridge to the classical neural activity since biochemical reactions mediated by free radicals are very sensitive to small changes of magnetic energies.
In Postulate (d), they propose how conscious experience emerges. Thus, they adopt a quantum state collapsing scheme from which conscious experience emerges as a set of collapses of the decoherence-resistant entangled quantum states. They further theorize that the unity of consciousness is achieved through quantum entanglements of these mind-pixels.

\subsection{Spin-Boson and Spin-Fermion Model of Consciousness}

Bosons, unlike fermions obey Bose-Einstein, who admits to a single quantum state could be an unlimited number of identical particles. Systems of many bosons described symmetric with respect to permutations of the particle wave functions.

Bosons differ from fermions, which obey Fermi-Dirac statistics. Two or more identical fermions cannot occupy the same quantum state (Pauli exclusion principle).

Since bosons with the same energy can occupy the same place in space, bosons are often force carrier particles. Fermions are usually associated with matter.

Fermions, unlike bosons, obey Fermi-Dirac statistics: in the same quantum state can be no more than one particle (Pauli exclusion principle).

\subsection{Topological Model of Consciousness}

It is known that the topological phase transition Kosterlitz-Thouless-phase transition in a two-dimensional XY-model. This transition is from the bound pairs of vortex-antivortex at low temperatures in a state with vortices and unpaired antivortices at a certain critical temperature.

$\mathrm{XY}$-model-a two-dimensional vector spin model which has symmetry $U$ (1). For this system is not expected to have a normal phase transition of the second order. This is because the system is waiting for the ordered phase that is destroyed by transverse vibrations, i.e. the Goldstone modes (see. Goldstone boson) associated with the breach of the continuous 


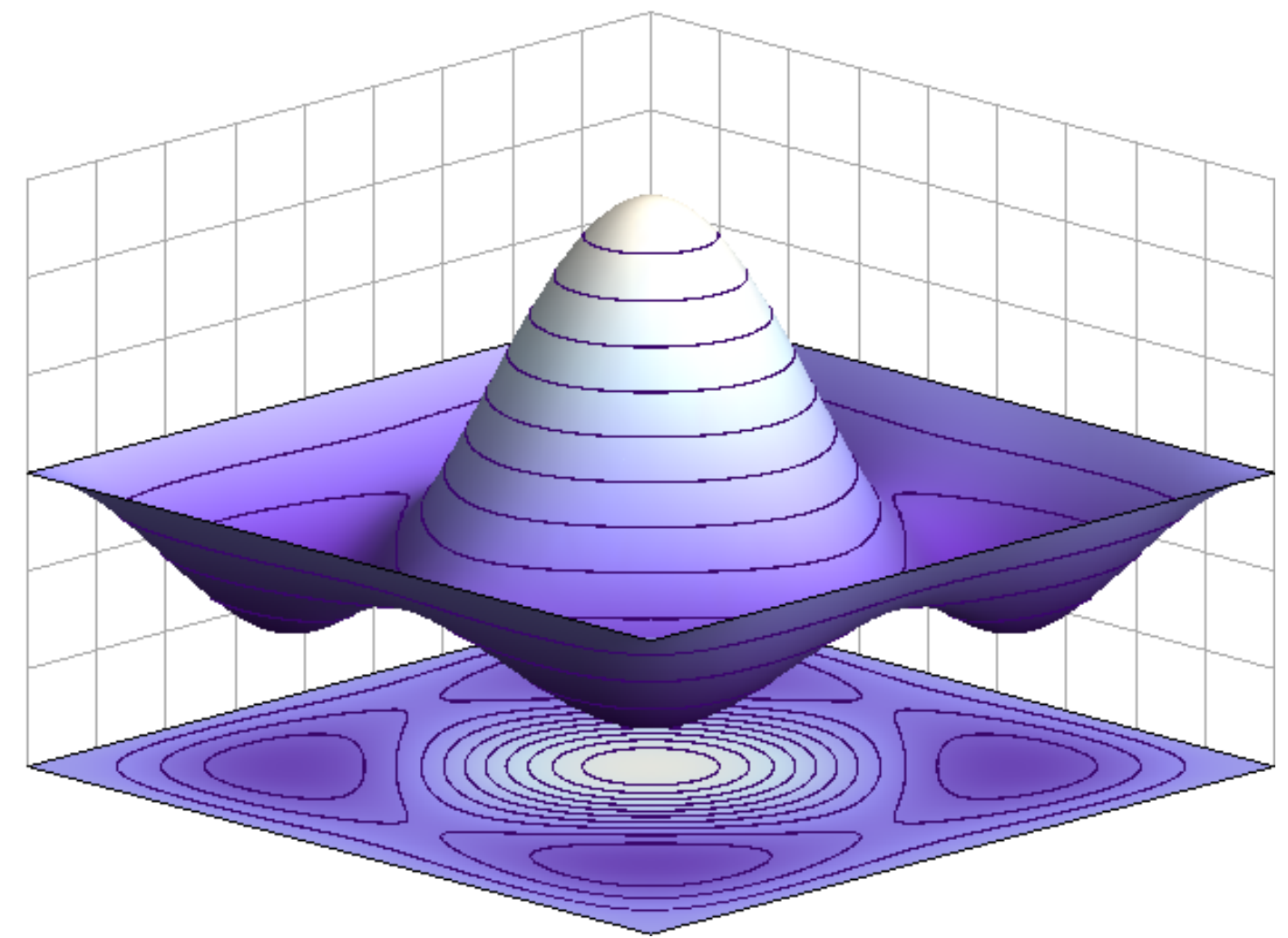

Fig. 1 Symmetric wavefunction for a (bosonic) 2-particle state in an infinite square well potential.

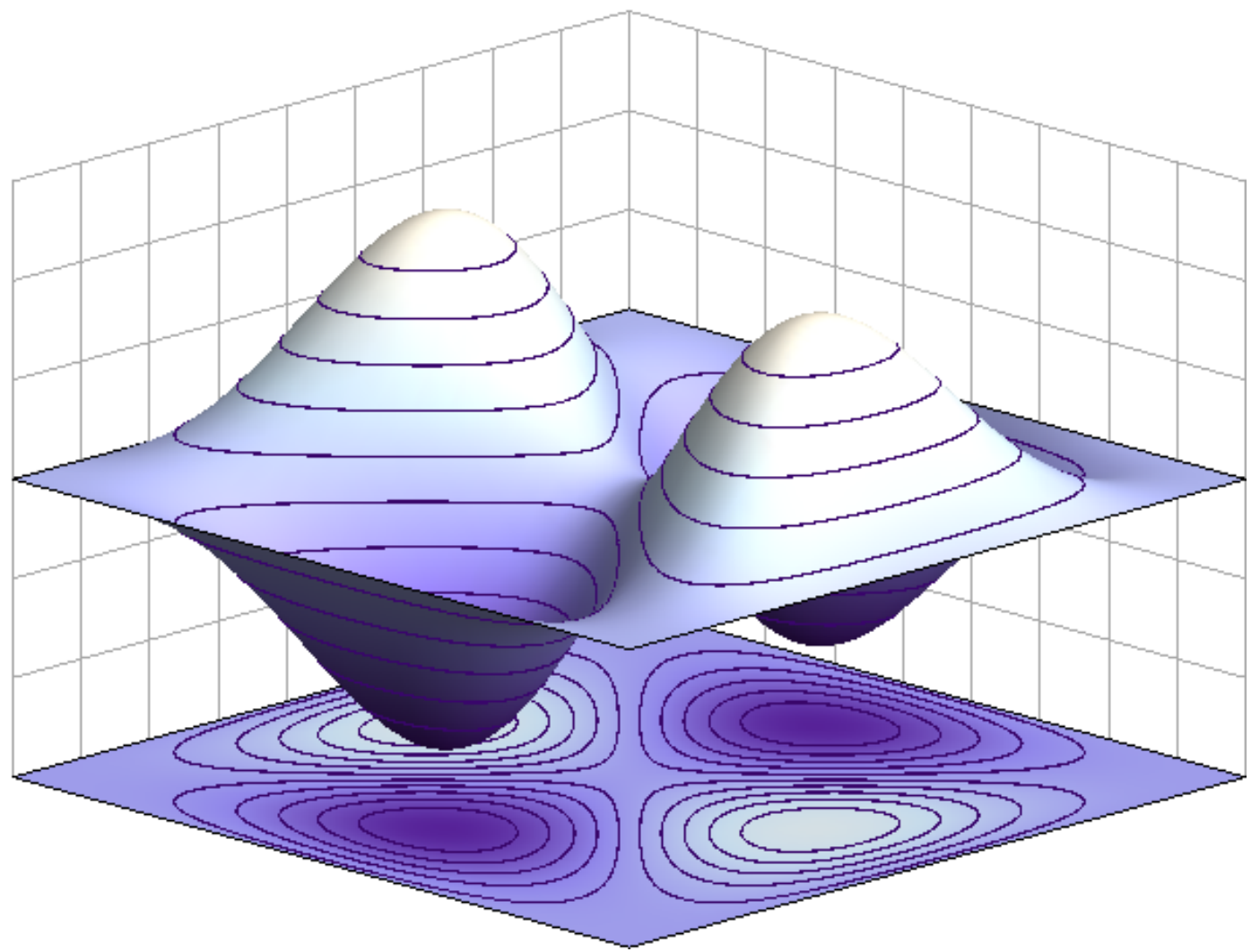

Fig. 2 Antisymmetric wavefunction for a (fermionic) 2-particle state in an infinite square well potential. 


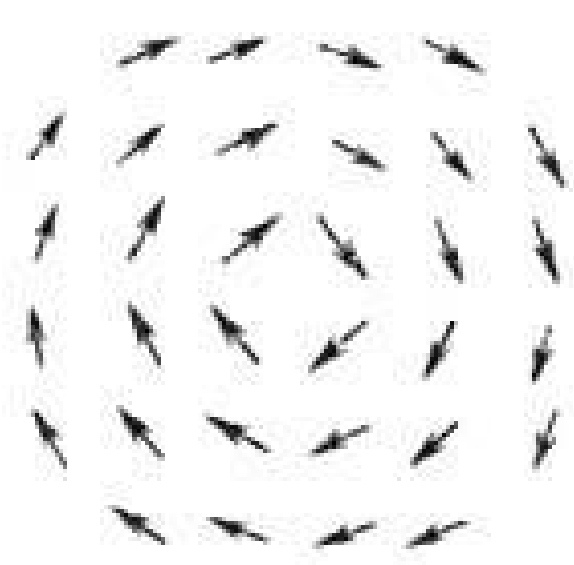

(a)

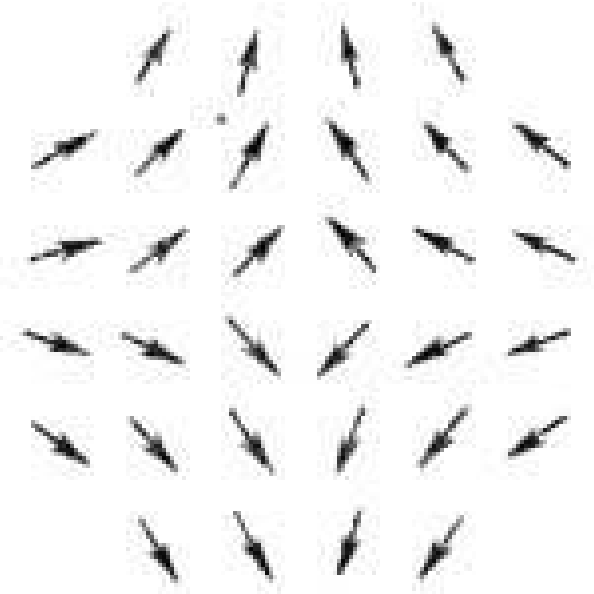

(b)

Fig. 3 Schematic image of a vortex (a) and antivortex (b) in the example of a planar magnetic material (arrows-vectors of the spin magnetic moments).

symmetry, which logarithmically diverge with increasing system size. This is a special case of Theorem Mermin- Wagner for spin systems.

Fig. 3 shows a schematic image of a vortex (a) and antivortex (b) in the example of a planar magnetic material (arrows - vectors of the spin magnetic moments).

Thus, the topology does not depend on the measurement of distances, it is so powerful. The same theorems are applicable to any complex symptom, regardless of its length or belonging to a particular species.

\section{Conclusions}

In conclusion, the authors have presented an alternative model of consciousness in which the unpaired electron spins are playing a central role as the mind pixels and unity of mind is achieved interweaving these mental pixels.

The authors hypothesized that these entangled electron spin states can be formed by the action potential modulated exchange and dipolar interactions, plus $\mathrm{O}_{2}$ and $\mathrm{NO}$ drive activations and survive rapid decoherence by quantum Zeno effects or decoherence-free spaces. Further, the authors have assumed that the collective electron spin dynamics associated with these collapses can have effects through the spin on the classic chemistry of neural activity, thereby affecting the neural networks of the brain. Our proposals involve the expansion of the associative neural coding of memories dynamic structures of neuronal membranes and proteins. Therefore, in our electron spin based on the model of the neural substrates of consciousness consists of the following functions: (a) electronic spin networks embedded in neuronal membranes and proteins, which serve as "crazy" screen with unpaired electron spins as pixels, (b) the nerve membrane and the proteins themselves, which serve as templates for the mind and nervous screen memories; and (c) free $\mathrm{O}_{2}$ and $\mathrm{NO}$, which serve as agents pixel activating.

Thus, the novelty of our work is that we were the first to propose that the electromagnetic field and free radicals have a great influence on the mind.

Thus, the authors have proposed a possible mechanism of the free radical $\mathrm{O}_{2}$ and $\mathrm{N}_{2} \mathrm{O}$ in the consciousness.

\section{References}

[1] Hu, H. P., and Wu, M. X. 2006. "Nonlocal Effects of Chemical Substances on the Brain Produced Through Quantum Entanglement." Progress in Physics 3: 20-6.

[2] Hu, H. P., and Wu, M. X. 2006 "Photon Induced Non-local Effects of General Anaesthetics on the Brain." Neuro Quantology 4 (1): 17-31. 
[3] Likhtenshtein, G. I. 1974. Spin labeling methods in molecular biology. John Wiley and Sons; London, New York, Sydney, Toronto.

[4] Likhtenshtein, G. I., Yamauchi, J., Nakatsuji, S., Smirnov, A., and Tamura, R. 2008. Nitroxides: Applications in
Chemistry, Biomedicine, and Materials Science. Cinii: Wiley.

[5] Aibassov, Y., Yemelyanova, V., and Savizky, R. 2016. "Magnetic Effects in Brain Chemistry." Journal of Chemistry and Chemical Engineering 10: 103-8. 